Review

\title{
Mitigation Techniques to Reduce the Impact of Wind Turbines on Radar Services
}

\author{
David de la Vega ${ }^{1, *}$, James C. G. Matthews ${ }^{2}$, Lars Norin ${ }^{3}$ and Itziar Angulo ${ }^{1}$ \\ ${ }^{1}$ Department of Communications Engineering, University of the Basque Country (UPV/EHU), Alda. \\ Urquijo s/n, Bilbao 48013, Spain; E-Mail: itziar.angulo@ehu.es \\ ${ }^{2}$ PA Consulting, Cambridge Technology Centre, Melbourn, Royston, Hertfordshire, SG8 6DP, UK; \\ E-Mail: jamescgmatthews@gmail.com \\ ${ }^{3}$ Swedish Meteorological and Hydrological Institute, Folkborgsvägen 17, Norrköping 601 76, Sweden; \\ E-Mail: lars.norin@smhi.se \\ * Author to whom correspondence should be addressed; E-Mail: david.delavega @ehu.es; \\ Tel.: +34-946-014127; Fax: +34-946-014259.
}

Received: 27 April 2013; in revised form: 29 May 2013 / Accepted: 30 May 2013 /

Published: 14 June 2013

\begin{abstract}
Radar services are occasionally affected by wind farms. This paper presents a comprehensive description of the effects that a wind farm may cause on the different radar services, and it compiles a review of the recent research results regarding the mitigation techniques to minimize this impact. Mitigation techniques to be applied at the wind farm and on the radar systems are described. The development of thorough impact studies before the wind farm is installed is presented as the best way to analyze in advance the potential for interference, and subsequently identify the possible solutions to allow the coexistence of wind farms and radar services.
\end{abstract}

Keywords: mitigation technique; impact study; weather radar; air traffic control radar; stealth technologies; data processing; adaptive clutter filters; adaptive scanning; gap filler radar

\section{Introduction}

The renewable energy sector has grown strongly and steadily for the last decade, and in particular wind energy has seen widespread adoption. More wind power capacity is installed and turbine 
manufacturers continue to increase average turbine sizes [1-3]. Wind farms are sometimes installed near radar sites, and this may cause degradation in the service provision, which may have an impact on safety or security.

In recent years, important research projects have been carried out, in order to characterize the signals scattered by the wind turbines and to determine the impact these reflected signals may cause on the detection capability of the radars. Additionally, a significant effort has also been put into developing various mitigation techniques, some of them based on the wind turbines design, but others in signal processing in the receiver or in the development of specific techniques for filtering the interfering signals.

The aim of this paper is to present a comprehensible description of the effects of the wind farm on the different radar services and to compile a review of the recent research results regarding the mitigation techniques to minimize this impact.

The structure of the paper is as follows: firstly, the effects of wind farms on the main types of radars are briefly described; secondly, the development of impact studies is proposed as the basic tool for analyzing the extent of the potential disturbance on the surrounding radars; and last, the different techniques for mitigating the impact are presented, classified into two groups, i.e., those applicable at the wind farm and techniques that require action by a third party.

\section{Effects of Wind Farms on Radars}

\subsection{Impact on Air Surveillance Radars}

The majority of work concerning the impact of wind farms on Air Surveillance Radars (ASR) has concentrated on Primary Surveillance Radar. Primary Surveillance Radars are typically S-Band radar systems operating in the $2.9-3.1 \mathrm{GHz}$ band. They provide range, bearing and velocity of non-cooperative air targets within several tens of kilometers.

The key parameters of interest in assessing the impact to ASR systems are reduction of probability of detection and production of false tracks and targets. For example, in a trial involving flying an Apache helicopter near a wind farm, the probability of detection was reduced by $6 \%-25 \%$ and $18-25$ false tracks were initiated per scan [4]. There are a number of ways these key parameters may be affected:

- Clutter: Unwanted echoes are considered as "clutter", and they may reduce the detection capability of the radar. The wind turbine presents a large physical target and therefore a large amount of energy is reflected back towards the radar. Much of the structure is static and therefore the return can be significantly reduced by conventional Doppler processing, although this may not entirely suppress the reflections. The blades rotate with large tip speeds, which provide a significant Doppler profile and will not be rejected [5].

- Range Sidelobe Effects: ASRs utilize pulse compression and suffer from the appearance of range sidelobes. Returns from large objects such as a wind turbine can leak in through the sidelobes, causing a smearing of the wind turbine return in range [6].

- Detection Shadowing: Constant False Alarm Rate (CFAR) processing uses a sliding window to provide a varying threshold with range based on the local clutter levels. Since the wind farm 
appears as a large clutter return, the threshold is raised, reducing radar detection sensitivity around the wind farm [7]. The threshold is also raised around the vicinity of the wind farm due to the CFAR window range extent. This effect, coupled with the range sidelobe effects mentioned above, causes detection shadowing around the wind farm, potentially a few kilometers away.

- Ghost Targets: The wind farm towers produce large specular returns and can create a multipath environment. It is reported that this can result in false "ghost" targets appearing [5].

- Operation: ASRs form the sensors within a safety critical system and are therefore highly regulated. There are currently a set of rules by which Air Traffic Controllers operate based on decades of experience. Although possible, any modifications or updates to the regulations are not desirable and may be extremely expensive [8]. Flight rules may be modified but this may be a lengthy and costly process. In some cases regulations will prohibit the placement of a wind farm. As an example, the regulations for the air traffic controller is that when providing Radar Advisory Service (RAS) in uncontrolled airspace, the aircraft should be guided such that there is a five nautical mile separation from any clutter that appears on the radar screen [8]. Placing a wind farm near the approach of an airport so that it causes clutter would require the controller to modify the regulations to provide RAS. This case is not always possible.

\subsection{Impact on Weather Radars}

Weather radars are used for monitoring precipitation and winds, as well as for detection and warning of hazardous events such as hailstorms, heavy rainfall, and severe wind conditions. Errors in weather radar data may be propagated to affect the output of numerical weather prediction and flood forecasting models.

Wind turbines located in line of sight of a weather radar can affect the radar's measurements and give rise to clutter, erroneous wind estimates, and blockage [9-11].

- Clutter from wind turbines is especially harmful for weather radars. Echoes from wind turbine towers have zero velocity and can therefore easily be significantly reduced by built-in clutter filters that suppress echoes with zero or low radial velocities. However, echoes from the turbines' rotating blades can have very large and variable velocities, escaping the suppression of the clutter filter. Wind turbine clutter downrange from wind turbines can be visible for tens of kilometers behind wind turbines $[9,11]$. Such clutter tails are believed to be caused by multiple scattering effects (scattering between multiple turbines and/or scattering between turbine and ground) $[9,12]$ and/or scattering from turbulent eddies in the wake of the wind turbine [13].

- Doppler weather radars not only measure the echo strength of their targets but also their radial velocities. The motion of the rotor blades of a wind turbine is interpreted by the radar as the wind speed, leading to erroneous wind estimates. Furthermore, the spectrum width of the velocity measurements (a measure of the wind turbulence) can also be affected by wind turbines.

- Blocking: Wind turbines located near a weather radar may block a substantial part of the radar's measurement region. However, blockage caused by wind farms is not always noticeable in radar 
reflectivity images. One reason for this is that precipitation echoes are usually not spatially homogeneous and the effect of blockage may therefore not be apparent. For longer accumulation periods the precipitation becomes more homogeneous and blockage becomes clearly visible.

\subsection{Impact on Marine Radars}

There have been some initial concerns about the impact of off-shore wind farms on marine radars, both on ground radars located in the coastline and on ship-borne radars. In the UK, for example, it is anticipated that the majority of wind energy will be derived from large, off-shore farms that can contain turbines with $60 \mathrm{~m}$ blades.

Marine radars do not include Doppler processing, and interference is primarily due to echoes from the turbines, which present high radar cross section (RCS) values at these frequencies. Consequently, echoes of small crafts within the wind farm can merge with strong echoes generated by the turbines when the craft pass close to the towers, making them invisible to radar observers or automatic plotting facilities. The severity of this effect depends upon the incident angle of the radar beam to the turbine [14].

The ship-borne radars are mobile, and therefore, it is not feasible to consider mitigation strategies such as gap-fillers. Marine navigational radars are low complexity/cost and the practicality of introducing advanced signal processing is considered unlikely [15]. In practical trials, however, it was found that despite the interference to the radar systems, it was possible for a trained mariner to navigate safely in and around the wind farm and identify other vessels [16].

\section{The Basic Approach: Analysis of the Potential Impact}

There are many stakeholders in the world who would like a single "golden bullet" solution to the wind turbine/radar service problem that could be applied to all scenarios and make the problem disappear. In reality, of course, it is not that simple. Each wind farm is unique in the layout, type and number of turbines, the surrounding terrain, the orientation and range with respect to the radar systems and the aircraft flight paths. Apart from the technical issues, of course there are also financial issues; the overall profitability of the site may make certain options more attractive than others, for example. Each case, therefore, requires detailed analysis to determine the impact of the wind farm on the radar system and the likely efficacy of potential mitigation options, and any agreed mitigation action is likely to be the output of negotiations with a number of stakeholders.

In the event of a potential objection, the first mitigation option should be to perform detailed modeling of the specific case, as numerical simulation is a useful and low cost method to ascertain how effective a particular solution may be.

The first step in the analysis is usually to estimate if the wind farm is in line-of-sight of the radar system, considering altimetry data, wind turbines dimensions and layout, which can be used for initial site optimization. The next step is to perform detailed modeling of the scenario, including technical specifications of the radar services and threshold values for evaluating the potential degradation of each service. The accurate estimation of the potential impact considering all the above-mentioned issues is a complex task that requires a software tool that manage all these data and algorithms [17-19]. As radars 
are stochastic systems, it is not practical to "turn-off" the effect of the wind farm but rather it is necessary to agree on a threshold level, below which the interference is considered insignificant.

The results of the analysis must provide numerical values regarding the clutter level generated by the turbines, as well as additional outcomes that help to modify the wind farm layout for minimizing the impact on the radar.

Radar processing blocks should also be considered, in order to ascertain if there is likely to be a significant impact to the radar performance. If the impact study indicates that there may be an interaction, the model can be used to test various mitigation options.

Figure 1 shows an example of such an analysis; the main beam of a scanning radar (green) explores the surrounding volume. The sections of the beam that will impinge on the turbines are shown in red. This type of results provides an evaluation of the signals backscattered off the wind turbines. The analysis allows for considering different locations of the turbines and/or lowering of the turbines in such a way that they do not disturb the proper operation of the radar.

Figure 1. Results of an impact analysis on a radar. The figure shows the beam of a scanning radar (green) and the part of the beam that is affected by the wind turbines (red) [18].

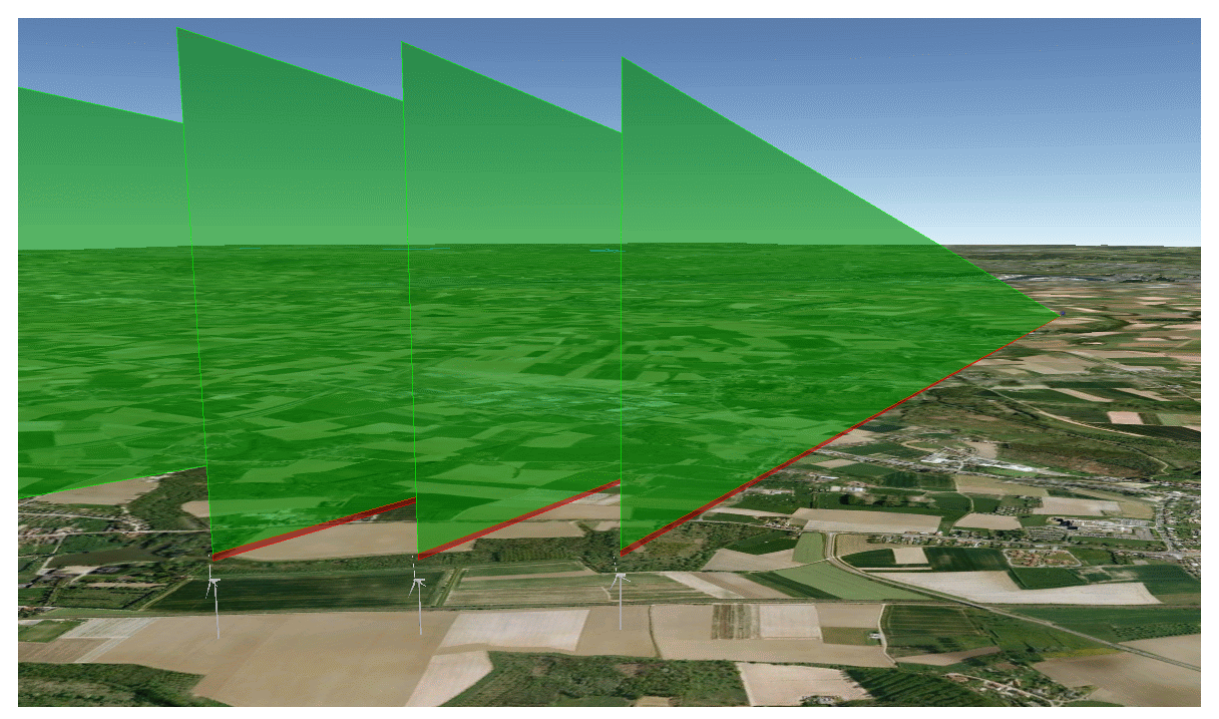

Figure 2 shows a different type of result of an impact analysis on a radar system. In this case, the red area is composed of the set of locations where a wind turbine will degrade the proper operation of the radar. For each particular location within the area under analysis, it is estimated if a wind turbine would generate a clutter signal above a specific level in the radar receiver, and in such case, the location is marked in red color. Consequently, the red mask is composed of the locations that should be avoided in the wind farm layout, in order to minimize the impact on the radar.

As a result, thorough impact studies may provide the necessary information for evaluating the extent of the impact and identifying the turbines and the candidate areas for the wind farm deployment that may cause interference, and accordingly, for including the modifications that may reduce the impact.

A key issue with this technique is the validation of any modeling results. The validation is difficult to achieve, due to the complex nature of the scenarios and the cost of conducting a trial, as radars are not typically available for experimental research and it is clearly prohibitively costly to build an 
experimental wind farm. Various trials have been conducted, but a concerted effort to amass sufficient data on the problem is needed.

Figure 2. Results of the impact analysis on a radar. Areas in red are composed by the sets of locations where a wind turbine would degrade the radar operation $[18,19]$.

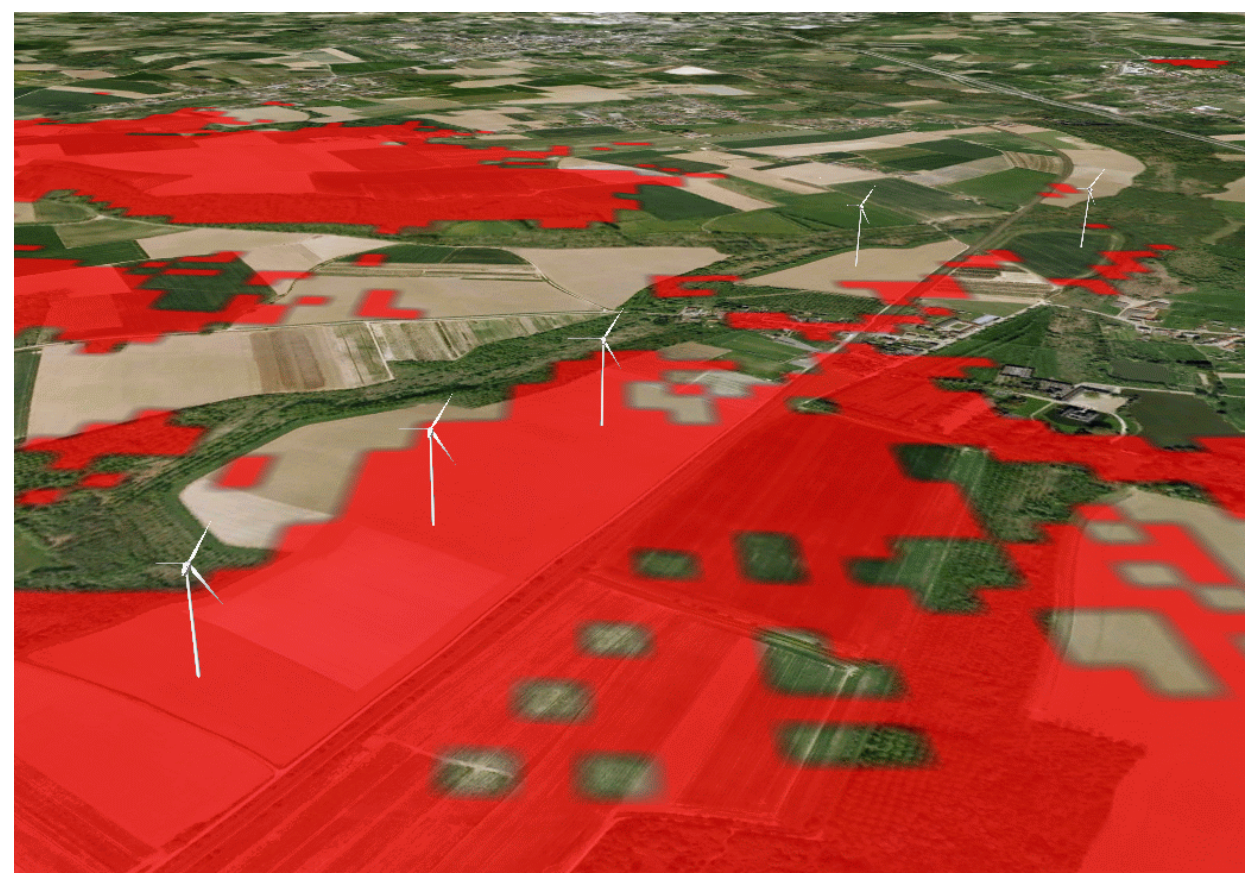

\section{Mitigation Techniques Associated to the Wind Farm}

\subsection{Guidelines Published by Regulatory Bodies}

Some regulatory bodies have published guidelines to estimate and avoid the impact of wind farms on radar services. Most of them aim to define rules-of-thumb and safeguarding zones that are easy to understand by a non-technical audience (from a radar perspective), such as wind farm developers.

Regarding the ASRs, Eurocontrol has published a document aimed at both providers of air navigation services and wind farm developers [7]. This document defines a number of zones and provides guidelines within each of these zones. These range from "safeguarding zones", within which no wind turbines should be placed, through zones requiring an impact assessment to be conducted, to zones in which no impact is expected. The first step in the assessment is to determine if any part of a turbine is within line-of-sight of the radar. If this is not the case, then it is stated that there will be no impact to the radar. The definition of these zones is reproduced in Figure 3. It should be noted that radar line-of-sight depends on atmospheric refraction, which at some locations may deviate from standard propagation conditions. It is therefore necessary to consider locally appropriate propagation conditions when assessing whether a wind turbine is in line-of-sight of the radar.

Guidance provided by the International Civil Aviation Organization states that an impact assessment should be conducted if a turbine is within $15 \mathrm{~km}$ of the radar [20]. The National Telecommunications and Information Administration also suggests the first step is to assess the line-of-sight of the radar and 
turbines, but also considers the effects of terrain shadowing, where diffraction of the radar signal can allow the radar to detect the turbine even for non-line-of-sight cases [21].

Figure 3. Zonal parameters for safeguarding distances and impact assessment (Source: [7]).

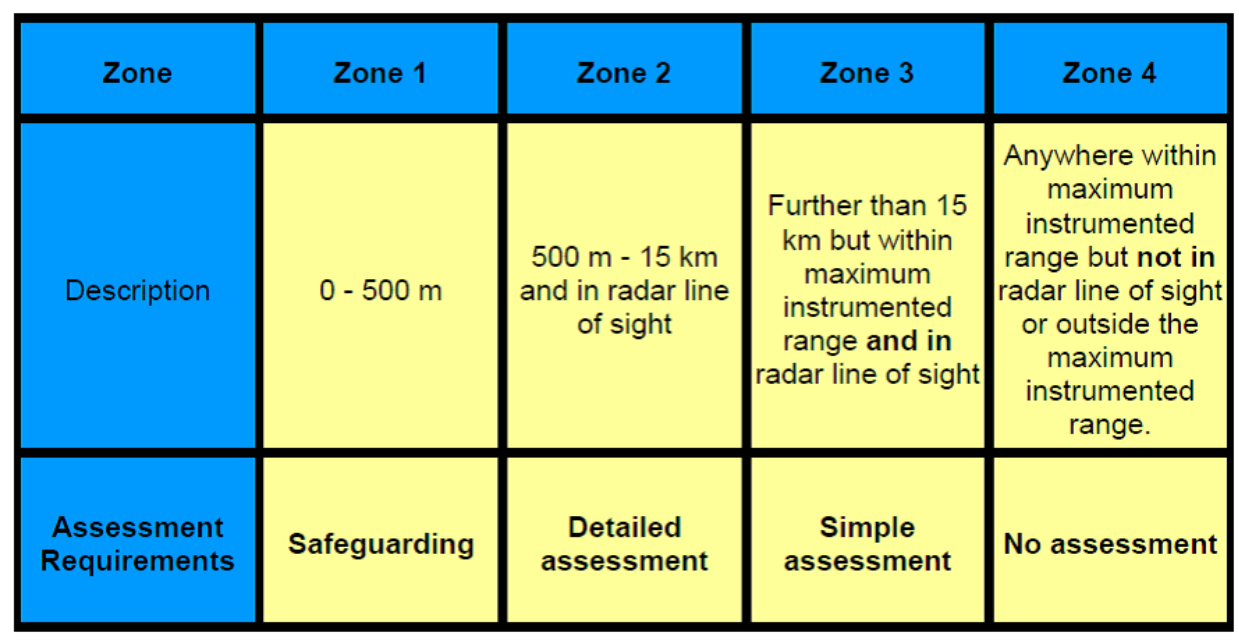

Both the World Meteorological Organization and the Network of European Meteorological Services have issued general guidelines for the deployment of wind turbines near weather radars, based on safeguarding distances [22,23]. According to these guidelines, no wind turbine should be deployed closer than $5 \mathrm{~km}$ to a weather radar, and wind farm developers are recommended to submit plans of wind farms located at a distance within $20 \mathrm{~km}$ from the radar for the development of an impact study. The International Telecommunications Union has also recognized the problem [13], but it has not yet issued any specific guidelines; only a recommended protection level for weather radars is stated, as an interference over noise level of $-10 \mathrm{~dB}$.

Although all the above-mentioned guidelines provide safeguarding distances and rules-of-thumb, they all propose the development of a case-by-case analysis, based on a detailed modeling of the scenario.

\subsection{Wind Farm Layout}

The wind farm layout could potentially be modified in some cases in order to reduce the impact to the radar system. This clearly needs to be achieved without affecting the viability of the wind farm. The effectiveness of such an option is dependent on terrain effects. For example, if a few turbines within a farm are within line-of-sight, removing those turbines may mitigate the problem. A typical rule adopted is that if the wind turbine is not within line-of-sight of the radar, it will not cause an impact on the radar [24]. Alternatively, increasing the spacing between wind turbines in a farm in such a way that they are individually resolvable will help with the detection of targets within the farm [24,25].

\subsection{Stealth Treatment}

Another option is the adoption of stealth technologies or stealth coating, as a technique for reducing the RCS of the turbines, and hence reduce the clutter generated by the wind turbines [25-27]. The RCS pattern of a wind turbine represents the power scattered by the wind turbine in all directions when a 
plane wave impinges on it [28]. The characterization of the RCS is a highly complex task, and there is currently a great effort in obtaining accurate and representative turbine RCS patterns [29]. Examples of measured and calculated RCS can be found in [24,30-32].

Stealth technology is well established in military world, as it has been studied extensively since the early days of radar. Stealth techniques are based, mainly, on modifying the object by shaping and coating with radar signal absorbing materials, in order to reduce the power scattered towards the radar antenna. Much of this research can be transferred into the civil domain and applied to wind turbines, although there are various constraints. First, a wind turbine represents a very large surface area, and therefore, the cost per square meter of stealth treatment must remain low. Additionally, any significant increase in mass of the turbine components is prohibitive due to the overall impact on the structure (for example, the impact on the gearbox loading due to the impact on blade mass).

Stealth technologies can be applied to the main elements of the turbine (mast, nacelle and blades) in a different way.

The wind turbine tower is typically an electrically large cylinder and provides a very directive, large radar return. The directivity, however, results in a large return only when the radar stares directly at the tower. In cases where this does not occur, the tower can remain unchanged. If the large return from the tower is received by the radar, much of the return will be significantly reduced due to the Doppler processing, which will attenuate the returns from a stationary object. Nevertheless, a radar system will not necessarily completely cancel a very large return from a stationary object, and some of the tower return may be detected [24]. In such case, shaping of the tower into a more conical shape may be an option to direct the specular return away from the radar. Application of radar absorbing materials on the mast is also an option, but it can be expensive, due to the large surface area and potential for increased service costs.

The nacelle can be treated with radar absorbing materials or shaping, depending on the cost benefit trade-off [25].

Most work in the literature on stealth treatments for turbines has concentrated on the blades. These are large objects, and their movement produces a large, non-zero Doppler return that can affect the radar [30,31]. The aerodynamic shape and the elevation angle of the blades mean that a time varying return is seen by the radar with a "flash" of high RCS for certain blade rotation angles. Stealth treatment for the blades is not trivial due to the blade structure and composition (they are made of various material layers incorporated to make a light, strong structure, and typically include some form of lightning protection, either a mesh or a rod), and because the blade shape is determined by aerodynamic factors. As an example, a $40 \mathrm{~m}$ turbine blade may cause RCS flashes of around $45 \mathrm{dBsm}$. Theoretically, stealth technologies may reduce these flashes by $15-20 \mathrm{~dB}$, still two orders of magnitude greater than a typical light aircraft, but recent studies only show a reduction of $10 \mathrm{~dB}$ [33]. The reason of this divergence comes from the fact that the stealth treatment is applied in a way that minimizes the changes of the existing blade design, which leaves very little room for the stealth material to be incorporated. A blade design that considers the radar material application from the beginning may well achieve better reductions in RCS. 


\section{Mitigation Techniques Associated to the Radar Services}

Mitigation options can be also applied to the radar services. The main options are adaptive clutter filters, the installation of gap filler radars, radar processing techniques, and the use of adaptive scanning in the radar antennas.

All these techniques are aimed to remove the clutter and ghost targets from the wind turbines. Echoes from wind turbines are a stochastic phenomenon, and therefore, the goal of completely removing this clutter and avoiding a reduction in the probability of detection is unrealistic. Nonetheless, these mitigation techniques have provided significant advances in the detection capacity in presence of wind farms.

\subsection{Data Processing in Air Surveillance Radars}

Various aspects related to the data processing in the reception chain of an ASR can be modified to reduce the effect of wind farms.

Early options were often applied in the post-detection stage (once the radar has determined the presence of the turbine). They include inhibiting track initiation in the vicinity of the wind farm or range-azimuth gating [34]. The main drawback of these options is that they will also inhibit the detection of wanted targets around the wind farm, and a "blind area" is generated around the wind farm area.

Pre-detection options are those applied to raw data, before the presence of an object is determined by the radar. They include the use of elevation beam information to discriminate higher altitude aircraft from lower altitude wind farms. This data information is included further along the processing chain. Additional techniques such as enhanced CFAR, moving target detector processing [34], high resolution clutter maps and plot/track filters [35] can be included for filtering the clutter signals out.

These signal processing options can be implemented using add-on hardware to modify the existing radar [35]. Nevertheless, it is likely that this option will require a recertification of the radar, which can incur significant costs.

\subsection{Adaptive Clutter Filters}

Adaptive clutter filters have been applied in weather radars to remove clutter signals from wind turbines. Such filters ideally identify the wind turbine signature, remove the corrupt measurements, and interpolate over the non-corrupt data to reconstruct the signal. The difficulty lies in identifying the wind turbine signature as it is time varying and highly complex. Furthermore, the wind turbine signature often resembles the actual weather signal. Nonetheless, several adaptive filter techniques for removing or reducing effects of wind turbine clutter have been suggested [10,12,36-40]. Such adaptive clutter filters can also help to mitigate erroneous wind measurements. If clutter is removed from signal, the average wind velocity as well as the spectrum width can easily be estimated.

Adaptive clutter filters use in-phase and quadrature phase (I/Q) measurements of the electric field as input. Since weather radars normally do not transmit I/Q data, but only the products based on it (reflectivity, radial velocity, etc.), the adaptive clutter filter should be implemented in the radar's 
signal processor. The main challenge of this mitigation technique is that it requires fast, reliable, and computationally effective filters.

To speed up filtering, only radar cells containing wind turbines should ideally be processed. This may be achieved by keeping maps of all wind turbines near a weather radar, or by using automatic detection schemes [41-44].

\subsection{Adaptive Scanning}

For weather radars, which primarily scan the sky for precipitation, the influence of wind turbines can be mitigated by adapting the scan strategy of the radar antenna. Changing the radar scan strategy to pass over areas with wind turbines could limit the amount of wind turbine clutter received and, therefore, reduce the undesired signal in the data processing. The drawback is that data obtained in the direction of the wind farm area would be gathered from higher altitudes, which may shorten the effective range of the radar.

A more advanced version of adapting the scanning strategy may be possible using phased array radars $[45,46]$. It has been suggested that the beam shape of the phased array radar can be altered in such a way that a null in the antenna radiation pattern is created in the direction of the wind turbine [47]. For such radars, this technique could provide an elegant way to reduce wind turbine clutter, but at the expense of a heavy computational cost.

\subsection{Sensor Fusion/Gap Filler Radars}

It is possible to employ more than one radar sensor to provide additional coverage where the probability of detection of the original radar has been reduced by the introduction of a wind farm, and combine the radar plots in a plot fusion process. The additional data may come from an existing radar system or through the deployment of a new radar.

In the case a new radar is deployed, it may be a relatively simple, low-cost radar, specifically designed to provide enhanced detection in such small regions [5]. This is often termed "Gap Filler" radar. For example, holographic radars have been proposed to achieve "unambiguous differentiation between aircraft and turbines" [48].

\section{Conclusions}

The wind farms may affect the proper operation and reduce the detection capability of the surrounding radars. Although some regulatory bodies have published some guidelines in order to avoid severe impacts, the interaction of wind farms with radar systems is extremely specific to the particular site, with a number of factors contributing to the problem and the implementation of any mitigation option. Therefore, the solution to the problem varies significantly on a case-by-case basis, and for this reason, although in some cases the definition of an exclusion zone may be accepted, it is not guaranteed that this is acceptable in other locations or for other kind of radars.

Consequently, simulating the entire scenario (wind farm, terrain, propagation conditions, radar system and radar functionalities) to a sufficient level of detail, although far from trivial, is currently the best 
approach to analyze the potential impact and to identify the possible solutions. Taking into account the costs involved and the implications to safety critical systems, any analysis method must provide accurate results with high confidence level.

There are several applicable mitigation options, all of which based on modifications to be applied at the wind farm or in the radar system. The options on wind farms rely on modifying wind farm layout and the use of stealth technologies in the turbine design; the techniques in the radar services consist of incorporating advanced filters and signal processing, adaptive scanning, or installing new radars that obtain unaffected data in the area around the wind farm.

In most cases it is not possible that the mitigation option completely removes any effects of the wind farm; any planning case that may impact a radar system will require careful analysis and negotiation with stakeholders. In practice, all these options should be considered as a set of tools available to minimize the potential impact, and therefore, negotiate planning applications.

Further research in this area is necessary to move forward in the coexistence of wind farms and radar services. Commissioning trials that gather and publish accurate measured data about the impact of radar systems in order to validate the various simulation tools is an essential step in providing confidence in the impact assessment approach. More accurate guidelines and recommendations are also needed, and they should be harmonized and accepted by all the involved partners in the wind farms deployment.

\section{Acknowledgements}

This work has been supported in part by the Spanish Ministry of Economy and Competitiveness (project TEC2012-32370) and by the Basque Government (SAIOTEK program).

\section{Conflict of Interest}

The authors declare no conflict of interest.

\section{References}

1. Schubel, P.J.; Crossley, R.J. Wind turbine blade design. Energies 2012, 5, 3425-3449.

2. Barambones, O. Sliding mode control strategy for wind turbine power maximization. Energies 2012, 5, 2310-2330.

3. Kabouris, J.; Kanellos, F.D. Impacts of large scale wind penetration on energy supply industry. Energies 2009, 2, 1031-1041.

4. Lute, C.; Wieserman, W. ASR-11 Radar Performance Assessment over a Wind Turbine Farm. In Proceedings of the 2011 IEEE Radar Conference, Kansas, MO, USA, 23-27 May 2011; pp. 226-230.

5. Thiel, A.; Schouten, M.W.; de Jong, A. Radar and Wind Turbines: A Guide to Acceptance Criteria. In Proceedings of the 2010 IEEE International Radar Conference, Arlington, VA, USA, 10-14 May 2010; pp. 1355-1361. 
6. Matthews, J.C.G.; Sarno, C.; Herring, R. Interaction of Wind Turbines with Radar Systems. In Proceedings of the 2008 Loughborough Antennas and Propagation Conference, Loughborough, UK, 17-18 March 2008; pp. 461-464.

7. Borely, M. Guidelines on How to Assess the Potential Impact of Wind Turbines on Surveillance Sensors; Technical Report EUROCONTROL-GUID-130; Eurocontrol: Brussels, Belgium, 2010.

8. Federal Aviation Administration. Section 5. Radar Separation. In Air Traffic Organization Policy JO 7110.65U; U.S. Department of Transportation: Washington, DC, USA, 2012.

9. Crum, T.; Ciardi, E.; Sandifer, J. Wind farms: Coming soon to a WSR-88D near you. Nexrad Now 2008, 18, 1-7.

10. Gallardo-Hernando, B.; Pérez-Martínez, F. Wind Turbine Clutter. In Radar Technology; Kouemou, G., Ed.; InTech: Rijeka, Croatia, 2009.

11. Norin, L.; Haase, G. Doppler Weather Radars and Wind Turbines. In Doppler Radar Observations-Weather Radar, Wind Profiler, Ionospheric Radar, and Other Advanced Applications; Bech, J., Chau, J.L., Eds.; InTech: Rijeka, Croatia, 2012.

12. Isom, B.M.; Palmer, R.D.; Secrest, G.S.; Rhoton, R.D.; Saxion, L.; Allmon, T.L.; Reed, J.; Crum, T.; Vogt, R. Detailed observations of wind turbine clutter with scanning weather radars. J. Atmos. Ocean. Technol. 2009, 26, 894-910.

13. International Telecommunication Union (ITU-R). Technical and Operational Aspects of Ground-Based Meteorological Radars. Recommendation ITU-R; Technical Report M.1849; International Telecommunication Union; Geneva, Switzerland, 2009.

14. Poirier, R. Technical Information and Coordination Process Between Wind Turbines and Radiocommunication and Radar Systems; Technical Report; Radio Advisory Board of Canada: Ottawa, Canada, 2009.

15. Rashid, L.; Brown, A. Impact Modelling of Wind Farms on Marine Navigational Radar. In Proceedings of the 2007 IET International Conference on Radar Systems, Edinburgh, UK, 15-18 October 2007; pp. 1-5.

16. MARICO Marine. Investigation of Technical and Operational Effects on Marine Radar Close to Kentish Flats Offshore Wind Farm; Technical Report, CCE5 No.1; Department for Transport: London, UK, 2007.

17. Turner, S.; New, C. Assessing the Impact of Wind Farms on Radar Systems. In Proceedings of the IET and Electronics Weekly Seminar on Electromagnetic Propagation in Structures and Buildings 2008, London, UK, 4 December 2008; pp. 69-83.

18. De la Vega, D.; Fernandez, C.; Grande, O.; Angulo, I.; Guerra, D.; Wu, Y.; Angueira, P.; Ordiales, J.L. Software Tool for the Analysis of Potential Impact of Wind Farms on Radiocommunication Services. In Proceedings of the 2011 IEEE International Symposium on Broadband Multimedia Systems and Broadcasting, Nuremberg, Germany, 8-10 June 2011; pp. 1-5.

19. Cascón, I.; Cascón, J.C.; Angulo, I.; de la Vega, D.; Guerra, D.; Wu, Y.; Arrinda, A.; Fernández, I.; Angueira, P. New Algorithms for Estimating the Impact of Wind Turbines on Telecommunication Services. In Proceedings of the 2013 International Conference on Renewable Energies and Power Quality, Bilbao, Spain, 8-10 April 2013. 
20. The European and North Atlantic Office of ICAO. European Guidance Material on Managing Building Restricted Areas; Technical Report, ICAO EUR DOC 015; International Civil Aviation Organization: Paris, France, 2009.

21. Lemmon, J.J.; Caroll, J.E.; Sanders, F.H.; Turner, D. Assessment of the Effects of Wind Turbines on Air Traffic Control Radars; Technical Report, TR-08-454; Department of Commerse, National Telecommunications \& Information Administration: Washington, DC, USA, 2008.

22. World Meteorological Organization (WMO). Commission for Instruments and Methods of Observation; Technical Report, WMO-No.1046; World Meteorological Organization, Helsinki, Finland, 2010.

23. OPERA. Statement of the OPERA Group on the Cohabitation between Weather Radars and Wind Turbines. Available online: http://www.knmi.nl/opera/opera3/OPERA_2010_14_Statement_on_ weather_radars_and_wind_turbines.pdf (accessed on 23 April 2013).

24. Poupart, G.J. Wind Farms Impact on Radar Aviation Interests; Technical Report, FES W/14/00614/00/REP; QinetiQ: London, UK, 2003.

25. Matthews, J.C.G. Stealth Solutions to Solve the Radar Wind Farm Interaction Problem. In Proceedings of the 2007 Loughborough Antennas and Propagation Conference, Loughborough, UK, 2-3 April 2007; pp. 101-104.

26. Pinto, J.; Matthews, J.; Sarno, C. Radar Signature Reduction of Wind Turbines. In Proceedings of the 3rd European Conference on Antennas and Propagation, Berlin, Germany, 23-27 March 2009, pp. 3886-3890.

27. Alves, M.A.; Folgueras, L.C.; Luiza, M.C. Reduction of the Radar Cross Section of a Wind Turbine. In Proceedings of the Microwave and Optoelectronics Conference, Natal, Brazil, 29 October-1 November 2011; pp. 551-555.

28. Knott, E.F.; Shaeffer, J.F.; Tuley, M.T. Radar Cross Section: Its Prediction, Measurement and Reduction; Artech House: Norwood, MA, USA, 1985.

29. Jenn, D.C. Radar and Laser Cross Section Engineering, 2 ed.; Education Series, American Institute of Aeronautics and Astronautics: Reston, VA, USA, 2005.

30. Matthews, J.C.G.; Lord, J.A.; Pinto, J. RCS Prediction for Stealthy Wind Turbines. In Proceedings of the European Conference on Antennas and Propagation, Nice, France, 6-10 November 2006.

31. Ohs, R.R.; Skidmore, G.J.; Bedrosian, G. Modeling the Effects of Wind Turbines on Radar Returns. In Proceedings of the 2010 Military Communications Conference, San Jose, CA, USA, 31 October-3 November 2010; pp. 272-276.

32. Kent, B.M.; Hill, K.C.; Buterbaugh, A.; Zelinski, G.; Hawley, R.; Cravens, L.; Tri-Van; Vogel, C.; Coveyou, T. Dynamic radar cross section and radar doppler measurements of commercial general electric windmill power turbines Part 1: Predicted and measured radar signatures. IEEE Antennas Propag. Mag. 2008, 50, 211-219.

33. Rashid, L.S.; Brown, A. Partial Treatment of Wind Turbine Blades with Radar Absorbing Materials (RAM) for RCS Reduction. In Proceedings of the 4th European Conference on Antennas and Propagation, Barcelona, Spain, 12-16 April 2010. 
34. Sergey, L.; Hubbard, O.; Ding, Z.; Ghadaki, H.; Wang, J.; Pondsford, T. Advanced Mitigating Techniques to Remove the Effect of Wind Turbines and Wind Farms on Primary Surveillance Radar. In Proceedings of the 2008 IEEE Radar Conference, Rome, Italy, 26-30 May 2008; pp. 1-6.

35. Perry, J.; Biss, A. Wind Farm Clutter Mitigation in Air Surveillance Radar. In Proceedings of the 2007 IEEE Radar Conference, Boston, MA, USA, 17-20 April 2007; pp. 93-98.

36. Gallardo, B.; Pérez, F.; Aguado, F. Characterization Approach of Wind Turbine Clutter in the Spanish Weather Radar Network. In Proceedings of the 5th European Conference on Radar in Meteorology and Hydrology, Helsinki, Finland, 30 June-4 July 2008.

37. Bachmann, S.; Al-Rashid, Y.; Bronecke, P.; Palmer, R.; Isom, B. Suppression of the wind farm contribution from the atmospheric radar returns. In Proceedings of the 26th Conference on Interactive Information and Processing Systems for Meteorology, Oceanography, and Hydrology, Atlanta, GA, USA, 18-21 January 2010.

38. Bachmann, S.; Al-Rashid, Y.; Isom, B.; Palmer, R. Radar and Windfarms-Mitigating Negative Effects through Signal Processing. In Proceedings of the The 6th European Conference on Radar in Meteorology and Hydrology, Sibiu, Romania, 6-10 September 2010; pp. 81-86.

39. Nai, F.; Palmer, R.; Torres, S. Wind Turbine Clutter Mitigation Using Range-Doppler Domain Signal Processing Method. In Proceedings of 27th Conference on Interactive Information and Processing Systems, Seattle, WA, USA, 23-27 January 2011.

40. Kong, F.; Zhang, Y.; Palmer, R. Wind Turbine Clutter Mitigation for Weather Radar by Adaptive Spectrum Processing. In Proceedings of the 2012 IEEE Radar Conference, Atlanta, GA, USA, 7-11 May 2012; pp. 471-474.

41. Hood, K.T.; Torres, S.M.; Palmer, R.D. Automatic Detection of Wind Turbine Clutter Using Doppler Spectral Features. In Proceedings of the 34th Conference on Radar Meteorology, Williamsburg, VA, USA, 5-9 October 2009.

42. Hood, K.; Torres, S.; Palmer, R. Automatic detection of wind turbine clutter for weather radars. $J$. Atmos. Ocean. Technol. 2010, 27, 1868-1880.

43. Gallardo-Hernando, B.; Pérez-Martínez, F.; Aguado-Encabo, F. Wind Turbine Clutter Detection in Scanning Weather Radar Tasks. In Proceedings of the 6th European Conference on Radar in Meteorology and Hydrology, Sibiu, Romania, 6-10 September 2010.

44. Cheong, B.L.; Palmer, R.; Torres, S. Automatic Wind Turbine Identification Using Level-II Data. In Proceedings of the 2011 IEEE Radar Conference, Kansas, MO, USA; 23-27 May 2011; pp. 271-275.

45. Yoshikawa, E.; Ushio, T.; Kawasaki, Z.; Yoshida, S.; Morimoto, T.; Mizutani, F.; Wada, M. MMSE beam forming on fast-scanning phased array weather radar. IEEE Trans. Geosci. Remote Sens. 2012, 51, 3077-3088.

46. Isom, B.; Palmer, R.; Kelley, R.; Meier, J.; Bodine, D.; Yeary, M.; Cheong, B.L.; Zhang, Y.; Yu, T.Y; Biggerstaff, M.I. The Atmospheric imaging radar: Simultaneous volumetric observations using a phased array weather radar. J. Atmos. Ocean. Technol. 2013, 30, 655-675. 
47. Palmer, R.D.; Le, K.D.; Isom, B.; Torres, S.M. Spatial filtering of wind turbine clutter using adaptive phased array radars. In Proceedings of the 25 th Conference on International Interactive Information and Processing Systems for Meteorology, Oceanography, and Hydrology, Phoenix, AZ, USA, 11-15 January 2009.

48. Aveillant. Why It Works. Available online: http://www.aveillant.com/why-it-works/ (accessed on 23 April 2013).

(C) 2013 by the authors; licensee MDPI, Basel, Switzerland. This article is an open access article distributed under the terms and conditions of the Creative Commons Attribution license (http://creativecommons.org/licenses/by/3.0/). 